\title{
Norois
}

Environnement, aménagement, société

226 | 2013

Commerce et développement local, déplacements urbains, valorisations agricoles, découpages

électoraux

\section{La France et ses paysans}

Jean-Pierre Houssel

\section{(2) OpenEdition}

\section{Journals}

Édition électronique

URL : http://journals.openedition.org/norois/4618

DOI : $10.4000 /$ norois. 4618

ISBN : 978-2-7535-2287-9

ISSN : $1760-8546$

Éditeur

Presses universitaires de Rennes

Édition imprimée

Date de publication : 30 mars 2013

Pagination : 107-108

ISBN : $978-2-7535-2285-5$

ISSN : 0029-182X

Référence électronique

Jean-Pierre Houssel, «La France et ses paysans », Norois [En ligne], 226 | 2013, mis en ligne le 30 mars 2013, consulté le 23 septembre 2020. URL : http://journals.openedition.org/norois/4618 ; DOI : https://doi.org/10.4000/norois.4618

(c) Tous droits réservés 


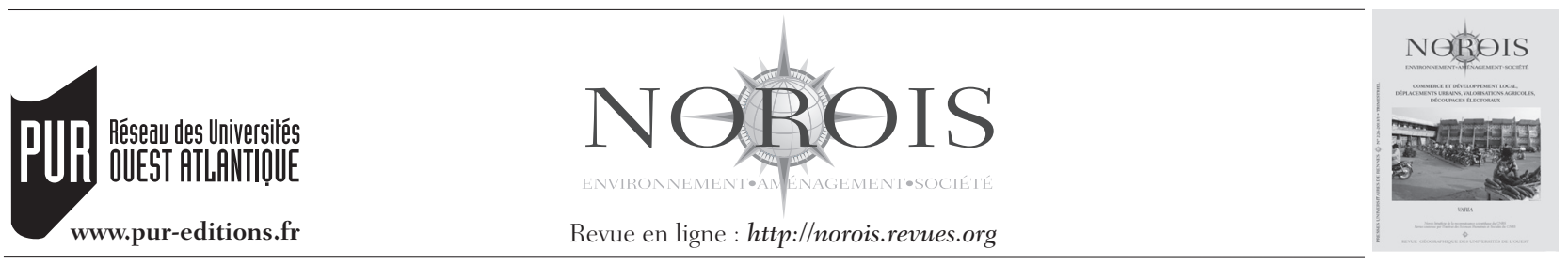

\section{COMPTES RENDUS BIBLIOGRAPHiQueS}

Emmanuel Laurentin, 2012. La France et ses paysans, Paris, Bayard/France-Culture, 219 p., $16,90 €$

Voilà un livre original du journaliste Emmanuel Laurentin, producteur de la série La Fabrique de l'Histoire, devenue quotidienne en 2004 sur FranceCulture. Il regroupe ici les six émissions consacrées à ce sujet entre 2002 et 2010 , dans un texte de 114 pages sous le titre De la méprise à la déprise, complété par cinq documents : tables rondes et entretiens. Il ne faut pas y rechercher une histoire classique et encore moins une géographie mais, comme l'indique le titre, la perception que le pays a de ses paysans et l'image qu'en donnent les médias.

La première partie consacrée aux belles années de la mutation de 1950 à 1970 est assez proche de ce qu'en disent les historiens : la prépondérance des agrariens dans les organisations professionnelles avant la guerre, un portrait classique de la période de Vichy nuancé par l'action des paysans dans la Résistance. Le rôle de l'INRA et de l'administration accompagnés par la recherche universitaire comme l'étude de Plozévet en Bretagne qui a révélé Edgar Morin est présenté comme majeur dans la révolution technique. En fait l'action des fils de paysans passés par la JAC (Jeunesse Agricole Catholique et non pas Chrétienne comme indiqué) est décisive dans son élaboration et son adoption dans les organismes conçus et gérés en commun comme les CUMA (pour les machines agricoles), les CETA (Centres d'Études Techniques Agricoles) et les groupements de producteurs. À travers le CNJA (Centre, et non pas Cercle National des Jeunes Agriculteurs) et avec l'entremise d'Edgard Pisani, ils sont les artisans de l'élaboration des Lois
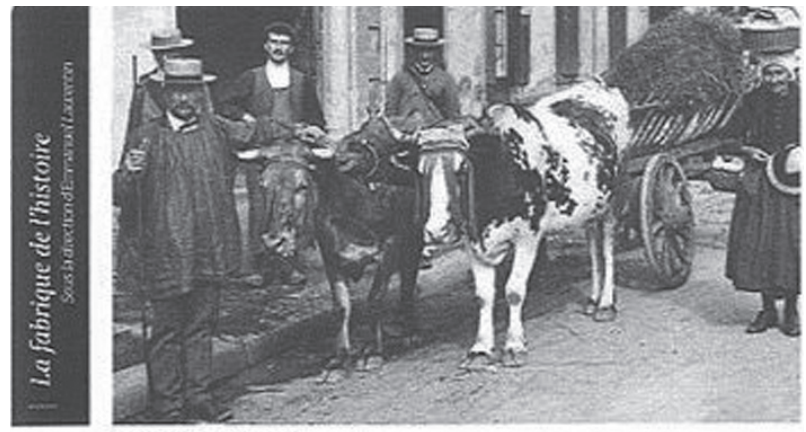

Emmanuel Laurentin
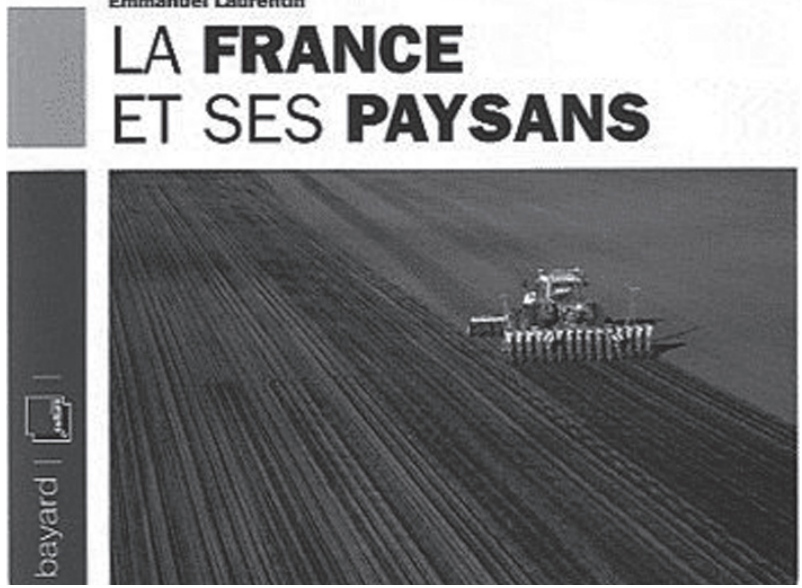

d'orientation agricole de 1960 et 1962 . Tout cela est d'ailleurs bien décrit. Mais la rédaction en courtes parties thématiques obère le rendu de la succession des phases du changement. On s'étonne que la révolution fourragère, innovation agronomique majeure, dont l'inégale adoption aurait permis de rendre 
compte des inégalités territoriales face à l'évolution ne soit pas signalée.

Pour la période postérieure qui conduit à la situation présente, les représentations et l'apparition de courants nouveaux prennent une place majeure, comme l'indique la succession des autres thèmes à côté des sujets économiques : la PAC toujours recommencée, la réussite agroalimentaire, la diminution rapide du nombre des exploitations. L'accent est porté successivement sur la contestation de la nouvelle FNSEA par les paysans-travailleurs de Bernard Lambert vers 1970, la gauche au pouvoir, l'arrivée des écologistes, le Larzac, les flamboyantes années de l'histoire rurale, le temps des récits des temps anciens, les femmes aussi (la promotion apportée par la JAC féminine), les divisions nouvelles (José Bové, la Confédération Paysanne), la représentation de l'abandon (Biquefarre et Depardon), le grand nombre de suicides d'exploitants, les questions d'appellation (la remise à l'honneur du terme paysan et le désarroi de la profession). La variété des approches montre qu'un regard extérieur est toujours utile aux spécialistes de nos disciplines.

Jean-Pierre Houssel

\section{Philippe Madeline et Jean-Marc Moriceau, 2012. Les paysans, récits, témoignages et archives de la France agricole (1870-1970), Paris, Les arènes, 112 p., 29,90 €}

Ce livre est le cinquième d'une nouvelle collection, l'Histoire entre nos mains, les précédents ayant été étant consacrés aux Français sous l'Occupation (1939-1945) en 2 tomes, à L'Algérie (1954-1962), à La France rouge à partir des archives du PCF (18711981). Ces beaux livres sont présentés sous étui et couvertures cartonnés, illustrés de photos couleurs. L'intérieur est réparti en titres constitués de deux pages se faisant face, où les textes laissent la plus grande place aux photos et cartes postales et ce qui en constitue l'originalité, à la reproduction de documents provenant d'archives publiques et surtout familiales sollicitées pour l'occasion. Ils sont soit simplement reproduits, soit ce qui est nouveau, disposés en feuillets intercalés ou détachables de leur étui. Ce sont disent les auteurs «des traces d'histoire pour la première fois devenues accessibles, archives vivantes et uniques entre nos mains ». Si le coût supplémentaire en main-d'œuvre n'est guère répercuté sur le prix de vente, c'est que le livre est imprimé en Chine.

Il allait de soi que le sujet des paysans souvent repris dans l'édition actuelle ne tarderait pas à être retenu. Il a été confié aux deux animateurs du Pôle rural de l'Université de Caen, reconnu de portée nationale. Le thème du changement sans précédent entre 1870 et 1970 s'imposait. Le découpage en périodes : 1870-1918, 1919-1945, 1946-1970 est bien choisi. Chacune est construite autour de son

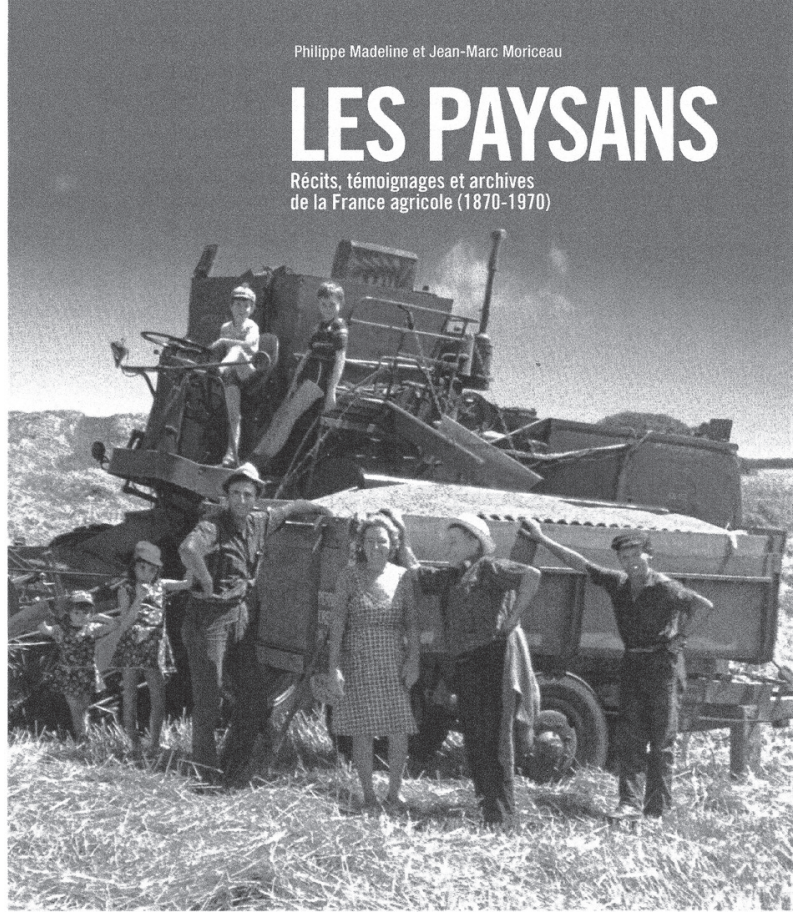

caractère dominant en une dizaine de centres d'intérêt. La première présente la prépondérance de la paysannerie à travers les attelages, l'importance du travail à la main, médailles et concours, la maison et la vie de tous les jours, mais aussi les apports de la République (l'école, l'arrivée des radicaux) et la catastrophe du phylloxéra. Trois doubles-pages sont 